\title{
Facial Numbness: A Manifestation of Sarcoidosis
}

\author{
Bobbi B. Adcock, $M D$
}

Facial numbness can pose a diagnostic dilemma for physicians. The trigeminal nerve can be affected by neoplasms in the nasopharynx, multiple sclerosis, dental and facial trauma, vertebrobasilar disease, carotid aneurysm, collagen vascular disease, infections including sinusitis and syphilis, and sarcoidosis. A careful history, physical examination, and prudent diagnostic studies are necessary to assess a patient with facial numbness.

Sarcoidosis, an idiopathic disorder characterized by noncaseating granulomas, has an incidence of 11 cases per 100,000 population in the United States. ${ }^{1}$ Sarcoidosis can affect one or many organs, including the lungs, lymph nodes, eyes, skin, heart, liver, and nervous system. According to most reports, the nervous system is affected in about 5 percent of patients with sarcoidosis. ${ }^{1-4}$ Sharma and Sharma, ${ }^{5}$ however, report that 10 percent of patients with sarcoidosis have nervous system involvement, and the finding of subclinical neurosarcoidosis is even greater. The case described below illustrates the importance of recognizing that facial numbness might be a manifestation of sarcoidosis.

\section{Case Report}

A 30-year-old African-American woman with a 5year history of pulmonary sarcoidosis came to the family medicine clinic complaining of a 2-week history of left facial numbness. In addition, she stated that the left side of her tongue felt like it had "beads" on it. She denied trauma; tick bites; occupational hazards; fever; headache; nausea and vomiting; seizures; dizziness or vertigo; visual changes; ear, nose, or throat problems; neck pain; weakness; or numbness elsewhere. She took $5 \mathrm{mg}$ of prednisone every other day for sarcoidosis until her gynecologist instructed her to discontinue it 4 months before this visit. The patient had a similar

Submitted 19 May 1998.

From the Department of Family Medicine, College of Community Health Sciences, University of Alabama School of Medicine-Tuscaloosa. Address reprint requests to Bobbi B. Adcock, MD, 700 University Blvd East, Tuscaloosa, AL 35401 . episode of numbness 11 months earlier that resolved spontaneously within 2 weeks. She had no other medical problems. Her only routine medication was oral contraceptives, and she was not allergic to any medications.

She was married and had 2 children, and she was a registered nurse. She had a family history of hypertension in her grandmother and prostate cancer and myocardial infarction in her grandfather. There was no family history of seizures, multiple sclerosis, neurofibromatosis, brain tumor, sarcoidosis, or collagen vascular disease.

During a physical examination her blood pressure, heart rate, respirations, and temperature were normal. Findings on examination of her skin, head, ears, eyes, nose, throat, neck, heart, and lungs were normal. Neurologically she had decreased sensation to light touch involving all branches of the left trigeminal nerve. Her other cranial nerves were intact. Her motor strength, peripheral sensory function, and deep tendon reflexes were normal.

Her facial neuropathy was thought to be secondary to sarcoidosis. The patient did not want to pursue any diagnostic studies, and prednisone 10 $\mathrm{mg} / \mathrm{d}$ was prescribed. At a follow-up examination 3 weeks later, she said that her left facial numbness had resolved after only 1 week of prednisone. In addition, she did not complain of the abnormal sensation of her tongue. Findings during a neurologic examination were normal. The prednisone dose was decreased to $5 \mathrm{mg} / \mathrm{d}$, which is her current regimen. She has had no adverse effects from the prednisone, and she has had no recurrence of the numbness.

\section{Discussion}

Sarcoidosis, because of its multisystem involvement, challenges the clinical acumen of all physicians. Sarcoidosis is more common in developed countries $^{6}$ and seems to be more severe in AfricanAmericans. ${ }^{6-8}$ Because the symptoms appear in African-Americans more abruptly, the likelihood of detection in this population might be increased. Chesnutt ${ }^{1}$ reports that sarcoidosis is found among African-Americans 10 times more often than in 
whites. The condition occurs most commonly between 20 and 40 years of age, and some studies have reported a slightly higher rate among women, but there is no clear sex predominance. ${ }^{6}$

Clinically the signs and symptoms of sarcoidosis are variable. In 90 percent of patients the respiratory tract is affected. ${ }^{7,8}$ Many patients report dyspnea with exertion, chest pain, and cough. Most patients have systemic complaints, including fatigue, fever, anorexia, and weight loss. Delaney ${ }^{3}$ reported that 65 percent of 23 patients had neurologic dysfunction as the initial finding of sarcoidosis. The dysfunction can include such entities as facial numbness or weakness, dysarthria, seizures, or myopathy. Sarcoid is the most likely cause of bilateral Bell's palsy in a patient. ${ }^{9}$ Sarcoid granulomas can affect any portion of the nervous system. Central nervous system involvement, which most commonly affects the cranial nerves, meninges, hypothalamus, and pituitary gland, usually occurs early in the disease. The peripheral nervous system is typically affected in the chronic stages of sarcoidosis. In this case, cranial nerve involvement occurred within 4 to 5 years of the diagnosis of sarcoidosis.

Almost one half of patients with neurosarcoidosis have more than one neurologic manifestation, ${ }^{2}$ with the facial nerve being the most commonly affected. ${ }^{3-5,9}$ Optic, glossopharyngeal, and vagus nerves are the next most frequently involved cranial nerves. When the trigeminal nerve is involved, there usually is unilateral sensory loss or neuralgia, and it is typically associated with other nerve palsies. Trigeminal involvement can be primary and not associated with other neurologic or systemic disorders, or it might be secondary to tumors, multiple sclerosis, vertebrobasilar disease, carotid aneurysm, infections, collagen vascular diseases, dental and facial trauma, and sarcoidosis. In our patient all three branches of the trigeminal nerve were involved, which caused numbness of her entire left face. Also affected was the facial nerve, which innervates the anterior two thirds of the tongue, resulting in the abnormal sensation of the tongue.

The diagnosis of neurosarcoidosis requires compatible clinical or radiologic evidence of sarcoidosis as well as histologic confirmation. If central nervous system involvement is suspected. a computed tomographic scan with contrast medium is the diagnostic procedure of choice, ${ }^{5}$ but magnetic resonance imaging is being used with increasing frequency. Other diagnostic tools include visual evoked potentials, brain stem auditory evoked potentials, and cerebrospinal fluid analysis including angiotensin-converting enzyme levels. The diagnosis of neurosarcoidosis in this patient was based on the history of sarcoidosis and clinical findings and was supported by her response to corticosteroids.

Corticosteroids are the mainstay of therapy for neurosarcoidosis. The usual dose of prednisone is 40 to $80 \mathrm{mg} / \mathrm{d}$ for cranial neuropathy, persistent peripheral neuropathy, and intracranial lesions. ${ }^{7}$ This dose can be prescribed for a few weeks and then tapered to the lowest maintenance dose. Prednisone may be discontinued after short-term therapy for patients who have aseptic meningitis or an isolated facial palsy. Long-term therapy is usually needed for intraparenchymal lesions, hydrocephalus, peripheral neuropathy, other cranial nerve involvement, or symptomatic myopathy. ${ }^{2}$ In some cases of neurosarcoidosis, surgery, radiotherapy, and immunosuppressive therapy might be necessary. The patient in this case responded to a much lower dose of corticosteroids than is recommended. She will be kept on a maintenance dose because two cranial nerves were affected.

The overall prognosis of sarcoidosis is good, as only 15 to 20 percent of patients have long-term functional impairment. ${ }^{1}$ Two thirds of patients with neurosarcoidosis have a self-limited monophasic illness, whereas one third have a chronic remitting-relapsing course. ${ }^{2}$ The prognosis is better for patients with peripheral nervous system compared with central nervous system involvement. ${ }^{3}$ Mortality for patients with neurosarcoidosis is higher than for patients without neurosarcoidosis. ${ }^{1}$ Stern et $\mathrm{al}^{4}$ reported that 55 percent of patients with neurosarcoidosis had complete recovery after corticosteroid therapy.

In summary, neurosarcoidosis should be considered in any patient who has sarcoidosis who complains of facial numbness. In fact, neurosarcoidosis should be considered in any patient with neurologic dysfunction; that dysfunction could be the initial clinical finding of sarcoidosis. Biopsies of cranial nerves are not commonly performed, so the physician must rely on clinical data, as in this case, and other diagnostic studies. Fortunately, most patients with neurosarcoidosis respond well to corticosteroids. 


\section{References}

1. Chesnutt AN. Enigmas in sarcoidosis. West J Med 1995;162:519-26.

2. Brust JCM. Neurosarcoidosis. In: Rowland LP, editor. Merritt's textbook of neurology. 9th ed. Media, Pa: Williams \& Wilkins, 1995.

3. Delaney P. Neurologic manifestations in sarcoidosis: review of the literature, with a report of 23 cases. Ann Intern Med 1977;87:336-45.

4. Stern BJ, Krumholz A, Johns C, Scott P, Nissim J. Sarcoidosis and its neurological manifestations. Arch Neurol 1985;42:909-17.
5. Sharma OP, Sharma AM. Sarcoidosis of the nervous system. A clinical approach. Arch Intern Med 1991; 151:1317-21.

6. Peckham DG, Spiteri MA. Sarcoidosis. Postgrad Med J 1996;72:196-200.

7. Newman LS, Rose CS, Maier LA. Sarcoidosis. N EnglJ Med 1997;336:1224-34.

8. DeRemee RA. Sarcoidosis. Mayo Clin Proc 1995; 70:177-81.

9. James DG. All that palsies is not Bell's. J R Soc Med 1996;89:184-7. 